\section{Acute psychotic presentations and acute psychosis}

We agree with Marneros (2006) that the concept of acute psychosis challenges the traditional Kraepelinian dichotomy. Clinical experience suggests that this condition is heterogeneous with the only similarity being the acuteness of onset. On longitudinal follow-up acute psychoses are separated into different categories; many resolve rapidly; some resolve only to recur again in a similar fashion; and others present differently over time to be reclassified as schizophrenia or mood disorders. It is therefore difficult to identify demographic or other characteristics that define this group.

The majority of patients who received this diagnosis in our study were young adults (mean age 29.75 years, s.d. $=10.95$ ) and male $(52 \%)$ (Thangadurai et al, 2006). This is in contrast to Marneros (2006) who reported a later age at onset and a predominance of women. A large group of our patients were later given a diagnosis of affective disorder $(9.2 \%)$, schizophrenia $(26.4 \%)$ or recurrent episodes of acute psychosis $(11.5 \%)$; others did not present with psychotic symptoms over the follow-up period. Although these results suggest that it is difficult to predict response to medication, course and outcome, it is well known that acuteness of onset is a good prognostic factor in both schizophrenia and mood disorders.

We argue that the concept of acute psychosis is necessary since many patients may present soon after the onset of illness, when the clinical features may not allow them to be categorised into any of the more classic disorders. Although many patients recover, some relapse with similar acute psychotic presentations; a significant proportion also develop schizophrenia and mood disorders. The difficulty in reaching a diagnosis at the initial presentation arises because it is often difficult to recognise the classic syndromes at this time. However, these can be identified over time. Thus, acute psychoses can be a presentation of the more traditional syndromes but can also be separate clinical entities which may or may not recur. Assuming that those who present with acute psychosis confirm to a homogeneous group does not fit with the clinical reality.

Marneros, A. (2006) Beyond the Kraepelinian dichotomy: acute and transient psychotic disorders and the necessity for clinical differentiation. British Journal of Psychiatry, 189, I-2.
Thangadurai, P., Gopalakrishnan, R., Kurian, S., et al (2006) Diagnostic stability and status of acute and transient psychotic disorders. British Journal of Psychiatry 188, 293.

\section{A. Kuruvilla, P. Thangadurai,}

\section{R. Gopalakrishnan, S. Kurien, K. S. Jacob}

Department of Psychiatry, Christian Medical College, Vellore, India 632002. Email: sanju@cmcvellore.ac.in doi: 10.II92/bjp.I89.6.565

Author's reply: Kuruvilla et al emphasise a point that we have also made in our study of acute and transient psychoses (Marneros \& Pillmann, 2004), namely the heterogeneity of 'acute psychoses' which are diagnosed only on the basis of acute presentation. Unfortunately, the ICD-10 diagnosis of acute and transient psychoses relies primarily on mode of onset and uses symptomatology only for sub-classification. As we have shown (Marneros \& Pillmann, 2004), a 'polymorphic', rapidly changing psychotic picture seems to be characteristic (as earlier authors always suggested) of the core group of acute polymorphous psychoses with good prognosis. In contrast, schizophrenia-like symptoms in the absence of polymorphic symptoms indicate a higher probability of later transition to schizophrenia. We believe that samples containing many of these patients with acute schizophrenia-like psychosis tend to lack the generally found female preponderance and show high rates of transition to schizophrenia early in the course. This is true for the sample of Amin et al (1999) and may also apply to that of Thangadurai et al (2006). Thus, although we agree with Kuruvilla et al about the heterogeneity of acute psychosis, we feel that further refinement of our diagnostic criteria might help better delineate the core group of acute polymorphous psychoses.

Amin, S., Singh, S. P., Brewin, J., et al (1999)

Diagnostic stability of first-episode psychosis. Comparison of ICD-10 and DSM-III-R systems. British Journal of Psychiatry, I75, 537-543.

Marneros, A. \& Pillmann, F. (2004) Acute and Transient Psychoses. Cambridge: Cambridge University Press.

A. Marneros Department of Psychiatry, Martin Luther University, 06097 Halle, Germany. Email: andreas.marneros@medizin.uni-halle.de doi: $10.1192 /$ bjp.189.6.565a

\section{Psychopathological consequences of ketamine}

Pomarol-Clotet et al (2006) reported a range of perceptual distortions in healthy volunteers following ketamine administration but did not report hallucinations. They concluded that ketamine does not reproduce the full picture of schizophrenia, but there are similarities in terms of referential thinking and negative symptoms.

We also recently studied healthy volunteers following ketamine administration (Stone et al, 2006) and, although previously unpublished, recorded phenomenological changes, including verbatim descriptions of their experiences. In keeping with Pomarol-Clotet $e t$ al, ketamine induced a wide range of abnormal perceptual experiences. However, no volunteers reported true hallucinations, although several reported eidetic imagery, and most reported visual illusions. Most experienced severe distortions of time, believing that a minute was several hours in duration. They also showed blunting of affect and loss of emotional reactivity. A few showed a marked disinhibition, with facetious replies to questions and apparent euphoria in the first 10 20 min after administration of ketamine. Several participants reported the belief that they were composed solely of thoughts, and that their bodies had either become nonexistent or were separate from them. One reported that he believed he could control people in the room by pointing with his hands, and another reported persecutory delusions.

Although we agree with PomarolClotet $e t$ al that these drug-induced effects do not correspond directly to schizophrenic symptoms, we feel it would be remarkable if ketamine administration were to completely reproduce the idiopathic condition. Ketamine induces a syndrome which is much closer to schizophrenia than other classes of psychotogenic substance, and, along with other NMDA receptor antagonists, is unique in inducing negative symptoms (Vollenweider \& Geyer, 2001). As ketamine has direct effects at receptors other than the NMDA receptor (Kapur \& Seeman, 2002), we believe that the next step should be to elucidate which particular receptors are responsible for each of the symptoms observed following ketamine administration. This may be achieved using similar analyses of psychopathology to those employed by Pomarol-Clotet et al combined with in vivo neurochemical imaging. 